\title{
Impressum, Vol. 9, No. 4, 1986
}

\section{s karger}

Verlag für Medizin und Naturwissenschaften GmbH, Postfach 1724, D-8034 Germering

Presserechtlich verantwortlich: Walter Kunz, Geschäftsführer und Gesellschafter

Wissenschaftlicher Beirat

H. W. Bauer, Berlin; W. Bossnew, Sofia/Bulgarien; H. Denck, Wien; V. Diehl, Köln; P. Drings, Heidelberg; E. Dühmke, Göttin-gen; S. Eckhardt, Budapest; H. Ehrhart, München; H. FE.

Fiebig, Freiburg; W. M. Gallmeier, Nürnberg; E. Fî. Graul, Marburg/ Lahn; R. Gross, Köln; R. Hartenstein, München; H. Heimpel, Ulm; K. P. Hellriegel, Berlin; Ch. Herfarth, Heidelberg; H. W. von Heyden, Einbeck; D. Hoelzer, Frankfurt/M.; J. H. Holzner, Wien; R. Hünig, Basel; W. Hunstein, Heidelberg; U. R. Klee-berg, Hamburg; H. O. Klein, Köln; B. Kornhuber, Frankfurt/M.; H. Löffler, Kiel; U. Mohr, Hannover; K. Munk, Heidelberg; G.A. Nagel, Göttingen; J. P. Obrecht, Basel; A. Pfleiderer, Freiburg; K. Possinger, München; W. Queißer, Mannheim; H. Riehm, Hannover; E. Scherer, Essen; M. Schroder, Göttingen; S. Seeber, Leverkusen; H. J. Senn, St.Gallen; A. Stacher, Wien; V. Sturm, Heidelberg; St. Tanneberger, Berlin/DDR; W.Vahlensieck, Bonn; H. D. Waller, Tubingen; M. Wannen-macher, Freiburg; W. Wilmanns, München; K. Wilms, Würzburg; K. zum Winkel, Heidelberg; H. Wrba, Wien. Schriftleitung: S. Eckhardt, Budapest J. H. Holzner, Wien G. A. Nagel, Göttingen Die Zeitschrift erscheint zweimonatlich; pro Jahr erscheint 1 Band zu je 6 Heften. Bezugspreis für Jahrgang 9, 1986 DM 148,-/öS 1036,-/SFr. 116,-. 1 Einzelheft kostet DM 28,/öS 196,-/SFr. 22,-, einschließlich MwSt., zuzüglich Postgebühren.

Der Abonnementsprcis ist im voraus zahlbar. Das Abonnement der Zeitschrift läuft weiter, wenn sie nicht spätestens 4 Wochen vor Abschluß eines Bandes abbestellt wird.

Abonnementsbestellungen können bei jeder Buchhandlung, bei der Post oder direkt beim Verlag aufgegeben werden: Bundesrepublik DeutschlandlÖsterreich: S. Karger GmbH, Postfach 1724, D-8034 Germering/München, Tel.: (089) 844021, Telex: 524865 D, Postscheckkonto: München 40080-807 Schweiz: S. Karger AG, Allschwilerstr. 10, Postfach, CH-4009 Basel, Tel: (061) 390880, Telex $62652 \mathrm{CH}$

Anzeigen

S. Karger Verlag für Medizin und Naturwissenschaften GmbH, Postfach 1724, D-8034 Germering, Telefon (089) 844021.

Gültig ist die Preisliste Nr. 4 vom 1.10.1982 (Einkleber auf Anfrage).

Für den Inhalt außerhalb des redaktionellen Teiles (insbesondere Anzeigen.

Industrieinformationen, Pressezitate und Kongreß-informationen) übernehmen Schriftleitung, Beirat und Verlag keine Gewähr.

Eine Markenbezeichnung kann warenzeichenrechtlich geschützt sein, auch wenn bei ihrer Verwendung in dieser Zeitschrift das Zeichen ${ }^{\circledR}$ oder ein anderer Hinweis auf etwa bestehende Schutz-rechte fehlen sollte. Für Satzfehler, insbesondere bei Dosierungs-angaben, wird keine Gewähr übernommen. 
Alle Rechte, insbesondere das Recht der Vervielfältigung und Mikrokopie sowie der Übersetzung, vorbehalten. Nachdruck, auch auszugsvveise, nur mit Genehmigung des Verlages. (C) Copyright 1986 by S. Karger Verlag für Medizin und Naturwissenschaften GmbH, Postfach 1724, D-8034 Germering

Satz und Druck: Walter Biering GmbH, Grafischer Betrieb, D-8000 München 45

Offizielles Organ

der Deutschen Gesellschaft für Hämatologie und Onkologie

und der

österreichischen Gesellschaft für Hämatologie und Onkologie

Offizielles Organ

der österreichischen Krebsgesellschaft - Krebsliga 